\title{
Original $\mid$ NEOADJUVANT DOCETAXEL AND CISPLATIN FOLLOWED BY CONCURRENT Article CISPLATIN WITH RADIATION THERAPY IN TREATMENT OF LOCALLY ADVANCED NASOPHARYNGEAL CARCINOMA
}

\author{
Ehab Hassan ${ }^{1}$ and Ahmed Fathi ${ }^{2}$.
}

${ }^{1}$ Department of Clinical Oncology, ${ }^{2}$ Department of E.N.T, Faculty of Medicine, Cairo University, Cairo, Egypt

\section{ABSTRACT}

\begin{abstract}
Aim of the Study: To assess the tolerability, toxicity and efficacy of neoadjuvant Docetaxel and Cisplatin (TC) followed by concurrent Cisplatin and radiotherapy (RT) in patients with locorgionally advanced nasopharyngeal carcinoma (NPC).

Patients and Methods: Fourteen patients with locally advanced NPC received two cycles of Docetaxel $\left(75 \mathrm{mg} / \mathrm{m}^{2}\right.$ on days 1, and 21) and Cisplatin ( $75 \mathrm{mg} / \mathrm{m}^{2}$ on days 1, and 21) followed by 7 weeks of Cisplatin ( $40 \mathrm{mg} / \mathrm{m}^{2}$ weekly) and RT at 70 Gy in 2-Gy fractions.

Results: Response to neoadjuvant TC was as follows: 5 patients (36\%) and 11 patients (79\%) achieved partial remission (PR) in regional nodes and nasopharynx, respectively. Nine patients $(64 \%)$ and 1 patient $(7 \%)$ achieved complete remission (CR) in regional nodes and nasopharynx, respectively. At 6 weeks after RT, 12 patients (86\%) achieved CR in regional nodes and nasopharynx, and 2 patients (14\%) achieved partial response (PR) in regional nodes and nasopharynx. Neoadjuvant TC was well tolerated. The most common acute toxicity of Cisplatin plus Docetaxel was grade 3-4 leucopenia (57\%) and alopecia (43\%). The most common acute toxicity of Cisplatin plus RT was grade 3-4 mucositis (64\%). At median follow-up of 12 months, one distant and one locorgional failure occurred.

Conclusions: Neoadjuvant Docetaxel and Cisplatin (TC) followed by concurrent Cisplatin and radiotherapy (RT) in patients with locorgionally advanced nasopharyngeal carcinoma (NPC) was feasible and resulted in excellent local tumour control with acceptable toxicity profile
\end{abstract}

Key Words: Nasopharyngeal carcinoma, neoadjuvant chemotherapy, radiotherapy

Corresponding Author: Ehab Ahmed Hassan, 123 Abd El-Aziz AlSaud, Manial, Cairo, Egypt

Tel. : 5080913, Mobile: 0123481619, E-mail: ehabhas@hotmail.com

\section{INTRODUCTION}

Radiotherapy is the primary treatment modality for all locally and regionally confined stages. Whereas the control of early-stage disease with radiotherapy is usually successful, the response of locally and regionally advanced NPC to radiation is poor because of local relapse and distant metastases. ${ }^{1-4}$

Diagnosis is made by biopsy of the nasopharyngeal mass. Workup includes careful visual examination (by mirror or endoscopic examination); documentation of the size and location of the tumour and neck nodes; evaluation of cranial nerve function and hearing; skull films (especially base of skull views), evaluating neural foramina; complete computed tomographic (CT) scan or magnetic resonance imaging (MRI) with views delineating the upper and lower extent of the lesion; chest $\mathrm{X}$-ray; hemogram; and chemistry panel. MRI is often more helpful than CT scans in detecting abnormalities and in defining their extent. ${ }^{5-7}$
Major prognostic factors adversely influencing outcome of treatment include large size of the tumour, higher $\mathrm{T}$ stage, and the presence of involved neck nodes. $^{8}$

The American Joint Committee on Cancer (AJCC) has designated staging by TNM classification to define nasopharyngeal cancer. ${ }^{9}$

Incorporation of chemotherapy with standard RT has improved the therapeutic outcome of patients with locoregionally advanced NPC. This is supported by a meta-analysis of six randomized trials suggesting that when compared with RT alone, the addition of chemotherapy in any sequence increases disease-free survival by $35 \%$ at 2 to 4 years and overall survival by $20 \%$ at 3 to 4 years $^{10}$. A key question remains regarding the optimal sequencing of chemotherapy and RT. Many published randomized trials of concurrent 
chemoradiotherapy have demonstrated a progressionfree (PFS) and/or overall survival (OS) benefit over RT alone ${ }^{11-13}$. In this regard, neoadjuvant chemotherapy may have better tolerability, and moreover impressive tumour response rates have been reported in phase II studies. ${ }^{14}$

\section{PATIENTS AND METHODS}

Fourteen patients with locally advanced nasopharyngeal carcinoma with no distant metastasis were enrolled in this study. All patients received two cycles of induction chemotherapy with Docetaxel $75 \mathrm{mg} /$ $\mathrm{m}^{2}$ and Cisplatin $75 \mathrm{mg} / \mathrm{m}^{2}$ on days 1 and 21 , followed by a radical course of radiotherapy (70Gy in 7 weeks, 200 cGy/ fraction) starting on Day 42, with concurrent Cisplatin $40 \mathrm{mg} / \mathrm{m}^{2}$ for 1 day on weekly basis till the end of radiation. All patients should have a non-metastatic disease as proved by doing chest and abdominal CT scan and bone scan. In this study, tumour staging was based on the 2002 American Joint Committee on Cancer (AJCC) (6th edition) criteria for NPC. ${ }^{9}$

\section{Inclusion criteria:}

- $\quad$ Age $>16$ years, $<75$ years.

- $\quad$ ECOG scale performance status of 0-2.

- $\quad$ Stage II, III or IV disease.

- At least one site of bi-dimensionally measurable tumour as assessed by clinical examination, computed tomography (CT) or magnetic resonance imaging had to be identified.

- Life expectancy of $>3$ months.

- $\quad$ No previous chemotherapy or radiotherapy prior to the study.

- Adequate hematopoietic reserve: absolute neutrophil count $>1.5 \times 109 / 1$, platelets $>100 \times 109 / 1$ with adequate hepatic and renal function: serum creatinine $<1.5$ times the upper normal limit (UNL), creatinine clearance $>60 \mathrm{ml} / \mathrm{min}$, serum bilirubin, AST and ALT $<1.5$ times UNL.

- All patients were required to provide signed informed consent.

\section{Exclusion criteria:}

Patients were excluded if they had received previous chemotherapy or radiotherapy. Patients who had active infections (including positive human immunodeficiency virus or unresolved hepatitis B patients), evidence of more than grade (1) peripheral neuropathy, or inadequate liver or renal functions. Also patients who were pregnant or breast feeding, or had a second malignancy or other serious concomitant medical disorders, were excluded from the study.

\section{Patient monitoring and follow up:}

Before treatment, patients underwent a complete history taking and physical examination, including evaluation of performance status, assessment for the presence of concurrent co-morbid conditions with estimation of weight, height, vital signs and measurement of palpable or visual tumour lesions. Laboratory studies included a complete blood count (CBC) with white blood cell differential count, urine analysis, biochemistry profile, kidney function test, liver function tests and glucose. Radiological examinations, including CT head and neck and CT scanning of chest, abdomen and pelvis, were required before starting treatment. Isotopic bone scan was not done routinely. Patients should have dental clearance before initiation of radiation therapy.

During therapy, performance status, weight, blood pressure, pulse rate, and full blood counts were obtained before each chemotherapy cycle. In addition, a complete medical history, including assessment of subjective (non-laboratory assessments) and objective (laboratory assessments) toxicity according to the WHO Common Toxicity Criteria (CTC) were obtained before the start of each new cycle. ${ }^{15,16}$

The primary end point of this study was response rate (RR). All patients had disease reassessment by physical examination, ENT examination and CT scan of the head \& neck. The treatment response was categorized according to Response Evaluation Criteria in Solid Tumours (RECIST). ${ }^{17}$

Six weeks after the completion of concurrent chemoradiation therapy; patients were assessed by clinical examination, full ENT evaluation and radiologically by CT scans.

\section{Chemotherapy protocol:}

Neoadjuvant chemotherapy comprised two cycles of Docetaxel $\left(75 \mathrm{mg} / \mathrm{m}^{2}\right.$ administered intravenously [IV] over 1 hour) given on days 1 and 21, followed by Cisplatin $\left(75 \mathrm{mg} / \mathrm{m}^{2}\right.$ administered intravenously [IV] over 1 hour) on day 1 and 21 repeated every 3 weeks. Docetaxel infusion was preceded by IV Dexamethasone $20 \mathrm{mg}$, Chlorpheniramine 50mg, Ranitidine 50mg and Gransetron $1 \mathrm{mg}$. All chemotherapy was given via a peripheral venous catheter in an outpatient setting. During RT, cisplatin at a dose of $40 \mathrm{mg} / \mathrm{m}^{2} \mathrm{IV}$ infusion was administered weekly for 7 weeks, given at approximately 60 minutes before receiving RT. Normal saline was given 
before $(500 \mathrm{~mL})$ and during (1 L) cisplatin infusion, during which mannitol was also used to maintain a high urine output.

\section{Radiation therapy technique:}

CT based planning was used. Phase I of radiation therapy to NPC would be carried out through 2 lateral head and neck fields including the nasopharynx and all surrounding tissues at risk of involvement taking into account to involve the primary tumour $+2 \mathrm{~cm}$ safety margin all around, the lateral neck nodes were included as well. The field setup would be 2 parallel-opposed beams with reference dose level at mid line. The lower neck and supraclavicular nodes were treated via a single anterior neck field. Radiation dose to lateral neck would be 40Gy given in 20 fractions over 4 weeks (200cGy per fraction), and the anterior neck field was given 50 Gy over 25 fractions in 5 weeks (200cGy per fraction).

Phase II was given by reduced lateral neck fields to include the nasopharynx and the surrounding tissues till the area just in front of the spine (off cord field) and radiation was given at a dose of $10 \mathrm{~Gy} / 5$ fractions / 5 days using 2 parallel opposed photon beams. Posterior neck nodes were boosted with an electron beam with variable energies dependent on depth of involved nodes, the dose given was $10 \mathrm{~Gy} / 5$ fractions / 1 week.

In Phase III (nasoparynx proper) the nasopharynx was treated as $20 \mathrm{~Gy} / 10$ fractions/ 2 weeks as 2 parallelopposed beams at midline using high photon energy.

\section{RESULTS}

From September 2005 to October 2006, 14 patients were enrolled onto this study. Patients and tumour characteristics are shown in table 1 . The mean age was 42 years with a range of 30-66 years. Twelve patients $(86 \%)$ were males, while 2 patients $(14 \%)$ were females. Performance status of ECOG (Eastern Cooperative Oncology Group) (0-1) and (2) was present in 10 patients $(71 \%)$ and 4 patients $(29 \%)$, respectively. Twelve patients (86\%) had WHO grade 2 histology. Eight patients (57\%) in the study presented with tumour stage T3, 5 patients (36\%) presented with T2 stage, 1 patient (7\%) with T4, and non of the patients presented with T1 lesion. Ten patients $(71 \%)$ presented with $\mathrm{N} 2$ disease, 2 patients (14\%) with N1 disease and 2 patients (14\%) with N3 disease. The main symptom at presentation was neck swelling which was present in $71 \%$ of cases followed by pain.

Overall, toxicity related to neoadjuvant Docetaxel and Cisplatin was manageable, table (2). Induction chemotherapy was generally well tolerated. A total of 28 cycles were given. All patients received 2 cycles of neoadjuvant chemotherapy. All 14 patients completed the planned treatment, and all were assessable for response and toxicity. During induction chemotherapy, most of the patients developed grade 1-2 WHO toxicity. Three patients $(21 \%)$ had a dose reduction of Docetaxel and

Table 1: Patients and Tumor Characteristics.

\begin{tabular}{|c|c|c|}
\hline Characteristic & No. & $\%$ \\
\hline $\begin{array}{l}\text { Age, years } \\
\text { Median } \\
\text { Range }\end{array}$ & \multicolumn{2}{|c|}{$\begin{array}{c}42 \\
30-66\end{array}$} \\
\hline Male: Female & $12: 2$ & $86: 14$ \\
\hline $\begin{array}{l}\text { ECOG performance status } \\
\text { ECOG }(0-1) \\
\text { ECOG }(2)\end{array}$ & $\begin{array}{c}10 \\
4\end{array}$ & $\begin{array}{l}71 \\
29\end{array}$ \\
\hline $\begin{array}{l}\text { WHO Histology } \\
\qquad \begin{array}{l}2 \\
3\end{array}\end{array}$ & $\begin{array}{c}2 \\
12\end{array}$ & $\begin{array}{l}14 \\
86\end{array}$ \\
\hline $\begin{array}{l}\text { Tumor stage T } \\
\text { T1 } \\
\text { T2 } \\
\text { T3 } \\
\text { T4 }\end{array}$ & $\begin{array}{l}- \\
5 \\
8 \\
1\end{array}$ & $\begin{array}{c}- \\
36 \\
57 \\
7\end{array}$ \\
\hline $\begin{array}{l}\text { Nodal stage N } \\
\qquad \text { N1 } \\
\text { N2 } \\
\text { N3 }\end{array}$ & $\begin{array}{c}2 \\
10 \\
2\end{array}$ & $\begin{array}{l}14 \\
71 \\
14\end{array}$ \\
\hline Metastasis M & - & - \\
\hline $\begin{array}{l}\text { Symptom at presentation } \\
\text { Neck swelling } \\
\text { Odynophagia } \\
\text { Nasal symptoms and epistaxis }\end{array}$ & $\begin{array}{c}10 \\
9 \\
9\end{array}$ & $\begin{array}{l}71 \\
64 \\
64\end{array}$ \\
\hline
\end{tabular}

Cisplatin. Five patients (36\%) had a dose delay of 5 days, most commonly because of delayed neutrophil recovery. Grade (1-2) anaemia occurred in 93\% of patients and only $7 \%$ of patients developed grade (34) anaemia. Grade 3-4 leucopoenia occurred in 57\% of patients, while grade (3-4) thrombocytopenia without bleeding occurred in $7 \%$ of patients. Two patients $(14 \%)$ developed uncomplicated, culture-negative grade 2 febrile neutropenia during induction chemotherapy. Grade (3-4) anorexia and nausea occurred in $7 \%$ of patients while, grade (3-4) vomiting occurred in $14 \%$ of patients. Grade $1-2$ sensory neuropathy occurred in $50 \%$ of cases. Forty three percent of patients developed grade (3 and 4) alopecia. Two patients (14\%) developed hypersensitivity reaction to Taxotere during the first cycle which was mild and manageable.

Concurrent Cisplatin with radiation was tolerable and of accepted toxicity profile. Most of the patients developed only grade (1-2) WHO hematologic toxicity. Nonhematologic toxicities related to Cisplatin, such as nausea, vomiting, and sensory neuropathy, were mild, and renal impairment was not encountered. 
The most commonly reported toxicity experienced during Cisplatin-RT was grade (3-4) mucositis within the RT field which occurred in $64 \%$ of cases. Grade (3-4) odynophagia occurred in $36 \%$ of patients. Grade (3-4) xerostomia and RT related skin toxicity were rare, while grade (1-2) was common (Table 3). Treatment-related deaths were not encountered in this study.

Table 2: Chemotherapy-Related Toxicity during Neoadjuvant Docetaxel/Cisplatin.

\begin{tabular}{lcccc}
\hline & \multicolumn{4}{c}{ WHO CTC Grade } \\
\cline { 2 - 5 } & \multicolumn{2}{c}{$\mathbf{1 - 2}$} & \multicolumn{2}{c}{$\mathbf{3 - 4}$} \\
\cline { 2 - 5 } & No. & \% & No. & \% \\
\hline Anemia & 13 & 93 & 1 & 7 \\
Leucopenia & 6 & 43 & 8 & 57 \\
Thrombocytopenia & 9 & 64 & 1 & 7 \\
Anorexia\& Nausea & 13 & 93 & 1 & 7 \\
Vomiting & 12 & 86 & 2 & 14 \\
Sensory Neuropathy & 7 & 50 & - & - \\
Alopecia & 8 & 57 & 6 & 43 \\
\hline
\end{tabular}

All patients showed response (CR or PR) to treatment both clinically and radiologically (Table 4 ). The overall response rate (CR and $\mathrm{PR}$ ) to neoadjuvant chemotherapy was $100 \%$ and $86 \%$ for lymph nodes and nasopharynx. After 6 weeks of neoadjuvant chemotherapy using Docetaxel and Cisplatin, 5 patients $(36 \%)$ achieved partial response (PR) and 9 patients (64\%) achieved complete

Table 3: Toxicity Related to Cisplatin-RT.

\begin{tabular}{lcccc}
\hline & \multicolumn{5}{c}{ WHO CTC grade } \\
\cline { 2 - 5 } & \multicolumn{2}{c}{$\mathbf{1 - 2}$} & \multicolumn{2}{c}{$\mathbf{3 - 4}$} \\
\cline { 2 - 5 } & No. & \% & No. & \% \\
\cline { 2 - 5 } Dysphagia & 14 & 100 & - & - \\
Mucositis within the field & 5 & 36 & 9 & 64 \\
Xerostomia & 13 & 93 & 1 & 7 \\
Odynophagia & 9 & 64 & 5 & 36 \\
Radiation Dermatitis & 13 & 93 & 1 & 7 \\
\hline
\end{tabular}

response (CR) in the regional nodes. Eleven patients (79\%) achieved PR and one patient (7\%) achieved CR in the nasophrynx. Six weeks post concurrent chemoradiation therapy; $86 \%$ of patients were in complete remission in regional nodes and nasopharynx.

With a median follow-up of 12 months, one patient developed distant metastases to bone, while the other developed locorgional failure (Table 5).
Table 4: Treatment outcome and response.

\begin{tabular}{llcll}
\hline Mode of Assessment & \multicolumn{2}{l}{$\begin{array}{l}\text { 6 Weeks Post } \\
\text { Induction } \\
\text { Chemotherapy }\end{array}$} & \multicolumn{2}{l}{$\begin{array}{l}\text { 6 Weeks Post } \\
\text { Concurrent } \\
\text { CRT }\end{array}$} \\
\hline & No. & \% & No. & \% \\
\hline LN, Clinical Examination & & & & \\
CR & 9 & 64 & 12 & 86 \\
PR & 5 & 36 & 2 & 14 \\
NPX, CT Scan & & & & \\
CR & 1 & 7 & 12 & 86 \\
PR & 11 & 79 & 2 & 14 \\
\hline
\end{tabular}

Abbreviations: CRT: Chemoradiation Therapy, LN: Lymph Node, NPX: Nasopharynx, CT: Computerized Tomography, CR: Complete Remission, PR: Partial Remission.

Table 5: Characteristics of 2 Patients who had Treatment Failure.

\begin{tabular}{lcc}
\hline Baseline TNM & Overall Response & Site of Relapse \\
\hline T3N2 & CR & Bone \\
T3N3 & PR & Nasopharynx and neck nodes \\
\hline
\end{tabular}

Abbreviations: TNM, Tumor-Node-Metastasis system

\section{DISCUSSION}

Patients with locorgionally advanced NPC have traditionally been treated solely with conventional RT; however, many develop local and/or distant failures and the long term survival rates are not satisfactory. In an attempt to improve treatment outcomes, several groups have incorporated chemotherapy adjuvantly, neoadjuvantly or concurrently with RT in randomized controlled trials. ${ }^{11-13,18-23}$ The results of a meta-analysis of 1528 patients from six randomized trials have shown that the addition of chemotherapy to radical RT for locorgionally advanced NPC increased both disease free/ progression free and overall survival by between 19 and $40 \%$ at $2-4$ years after treatment. ${ }^{10}$

The primary rationale for induction or adjuvant chemotherapy in nasopharyngeal cancer has been to decrease the risk of developing distant metastases. Promising 4- to 5-year OS rates exceeding 75\% have been reported in studies from Australia and North America using sequential neoadjuvant chemotherapy and chemoradiotherapy. Rischin et al..$^{24}$ used three cycles of the Cisplatin, Epirubicin, and infusional Fluorouracil (ECF) followed by concomitant RT at 60 Gy in 2-Gy fractions and Cisplatin (administered daily for 5 days in week 1 and 6). In another study, Hong et al. ${ }^{24}$ used three cycles of induction chemotherapy with Mitomycin, Epirubicin, Cisplatin, Fluorouracil, and Leucovorin (MEPFL) before RT. 
The three large trials of induction without concurrent chemotherapy have not demonstrated improved overall survival compared with radiation alone. ${ }^{25-28}$ Chan et al. used induction chemotherapy of 2 cycles of Taxol (Paclitaxel) and Carboplatin followed by concurrent Cisplatin RT. ${ }^{28}$ Chan et al. used induction chemotherapy of 2 cycles of Taxotere (Docetaxel) and Cisplatin followed by concurrent Cisplatin RT. ${ }^{29}$

In our study induction chemotherapy was generally well tolerated and of acceptable toxicity profile. Seven percent of patients developed grade (3-4) anaemia. This figure concurs with what had been reported by Rischin et al. and Wee et al. ${ }^{21,30}$ who reported grade (3-4) anaemia of $0 \%$ and $3 \%$, respectively. Grade (3-4) leucopenia occurred in $57 \%$ of patients. Our results are comparable to that of Hong et al. ${ }^{24}$ who reported an incidence of $59 \%$ of grade (3-4) leucopenia in his study. This finding is slightly higher than that reported by Rischin et al and Chan et al. ${ }^{21,28}$ who reported an incidence of grade (3-4) leucopenia of $19 \%$ and $36 \%$, respectively. This could be explained by the low myelotoxic potential of the regimen they used. Our results are much less than that reported by Chan et al. ${ }^{29}$ who reported an incidence of grade (3-4) leucopenia of $100 \%$ although they used the same regimen of induction chemotherapy. Grade (34) thrombocytopenia occurred in $7 \%$ of patients which coincides with Chan et al. and Wee et al..$^{29,30}$ who reported an incidence of grade (3-4 )thrombocytopenia of 1.4\% and $3 \%$, respectively. None of the patients experienced grade (3-4) febrile neutropenia or sensory neuropathy which again concurs with the results of Chan et al. ${ }^{28}$ Grade (3-4) vomiting occurred in 14\% of our patients which was slightly higher than what is reported by Chan et al. ${ }^{29}$ who reported a figure of $7 \%$. Forty three percent of our patients developed grade (3-4) alopecia which is slightly higher than what is reported by Rischin et al. ${ }^{21}$ who reported incidence of grade (3-4) alopecia of 31\%. Furthermore Chan et al. ${ }^{28}$ did not report any grade (3-4) alopecia in his study.

Chemotherapy related toxicity during concurrent Cisplatin with radiation was comparable to that reported by Chan et al ${ }^{28}$. In our study $64 \%$ of our patients developed grade (3-4) mucositis within the radiation field which concurs with the results of Chan et al. and Lee et al. ${ }^{28,29,31}$ who reported an incidence of $55 \%$ and $62 \%$ of grade (34) mucositis, respectively. Our results are higher than what had been reported by Rischin et al. ${ }^{24}$ who reported an incidence of $31 \%$ of grade (3-4) mucositis.

The current study confirms the feasibility of combining neoadjuvant TC (Taxotere- Cisplatin) followed by concurrent chemoradiotherapy in patients with advanced NPC and resulted in a complete remission rate (CR) in $86 \%$ of patients. Patient tolerability and compliance to neoadjuvant TC was good, and there were no treatment- related deaths. The outpatient administration of TC also avoids the need for hospital admission or indwelling catheters.

Tumour response in the regional nodes and nasopharynx were studied separately. The response rates (CR and PR) to neoadjuvant Taxotere and Cisplatin in the regional nodes and nasopharynx was $100 \%$ and $86 \%$, respectively. Chan et al. ${ }^{28}$ reported an overall tumour response in the lymph nodes and nasopahrynx of $97 \%$ and $16 \%$ respectively. This apparently differential response may be partially attributed to inadequacy of endoscopic and CT examination in evaluating the nasopharynx. Although CT scan is good in assessing bony structures of the head and neck and most nodal groups in the neck, it does not surpass MRI in assessing the retropharyngeal nodes or the soft tissue structures around the NP (especially parapharyngeal disease). ${ }^{32}$ Chan et al. $^{29}$ reported an overall response rate to neoadjuvant chemotherapy of $80 \%$ and $86 \%$ in the regional nodes and nasopharynx, respectively. Hong et al. ${ }^{24}$ reported complete remission rate of $45.5 \%$ and $6.4 \%$ in the regional lymph nodes and nasopharynx, respectively.

In our study, 6 weeks post concurrent chemoradiation $86 \%$ of patients were in complete remission in the regional lymph nodes and nasopharynx. Chan et $\mathrm{al}^{28}$ reported complete remission rates of $97 \%$ and $100 \%$ in the regional lymph nodes and nasopharynx, respectively. Chan et al. ${ }^{29}$ reported complete remission rate of $80 \%$ and $96 \%$ in the regional lymph nodes and nasopharynx, respectively. Hong et al. ${ }^{24}$ reported a complete remission rate of $67 \%$ in his trial

\section{CONCLUSION}

Neoadjuvant Docetaxel and Cisplatin (TC) followed by concurrent Cisplatin-RT used for patients with locally advanced nasopharyngeal cancer results in excellent tumour control and manageable toxicity profile

\section{REFERENCES}

1. Perez CA, Devineni VR, Marcial Vega V, Marks JE, Simpson JR, Kucik N. Carcinoma of the nasopharynx: Factors affecting prognosis. Int.J.Radiat.Oncol.Biol.Phys. 1992;23(2):271-80.

2. Zhang EP, Lian PG, Cai KL, Chen YF, Cai MD, Zheng XF, et al. Radiation therapy of nasopharyngeal carcinoma: Prognostic factors based on a 10-year follow-up of 1302 patients. Int.J.Radiat. Oncol.Biol.Phys. 1989 Feb;16(2):301-5.

3. Qin DX, Hu YH, Yan JH, Xu GZ, Cai WM, Wu XL, et al. Analysis of 1379 patients with nasopharyngeal carcinoma treated by radiation. Cancer 1988 Mar 15;61(6):1117-24.

4. Lee AW, Poon YF, Foo W, Law SC, Cheung FK, Chan DK, et al. Retrospective analysis of 5037 patients with nasopharyngeal 
carcinoma treated during 1976-1985: Overall survival and patterns of failure. Int.J.Radiat.Oncol.Biol.Phys. 1992;23(2):261-70.

5. Mendenhall WM, Riggs CEJ, Cassisi NJea. Treatment of head and neck cancers. In: DeVita VT, Hellman S, Rosenberg SA, editors. Cancer: Principles \& practice of oncology. 7th ed. : Lippincott Williams \& Wilkins; 2004. p. 662-732.

6. Laramore GE. Radiation therapy of head and neck cancer (Medical radiology diagnostic imaging and radiation oncology): Springer; 1989

7. Fredrickson JM, Harker LA, Krause CJ, Richardson M, Schuller DE, Cummings CW, et al. Otolaryngology: Head \& Neck Surgery. 3rd ed. : Mosby-Year Book; 1998.

8. Sanguineti G, Geara FB, Garden AS, Tucker SL, Ang KK, Morrison WH, et al. Carcinoma of the nasopharynx treated by radiotherapy alone: Determinants of local and regional control. Int.J.Radiat.Oncol.Biol.Phys. 1997 Mar 15;37(5):985-96.

9. In: Greene FL, Page DL, Fleming ID, Fritz A, Balch CM, Haller DG, et al, editors. AJCC Cancer Staging Manual. 6th ed. New York: Springer; 2002. p. 31-46.

10. Huncharek M, Kupelnick B. Combined chemoradiation versus radiation therapy alone in locally advanced nasopharyngeal carcinoma: Results of a meta-analysis of 1,528 patients from six randomized trials. Am.J.Clin.Oncol. 2002 Jun;25(3):219-23.

11. Al Sarraf M, LeBlanc M, Giri PG, Fu KK, Cooper J, Vuong T, et al. Chemoradiotherapy versus radiotherapy in patients with advanced nasopharyngeal cancer: Phase III randomized Intergroup study 0099. J.Clin.Oncol. 1998 Apr;16(4):1310-7.

12. Chan AT, Teo PM, Ngan RK, Leung TW, Lau WH, Zee B, et al. Concurrent chemotherapy-radiotherapy compared with radiotherapy alone in locoregionally advanced nasopharyngeal carcinoma: Progression-free survival analysis of a phase III randomized trial. J.Clin.Oncol. 2002 Apr 15;20(8):2038-44.

13. Lin JC, Jan JS, Hsu CY, Liang WM, Jiang RS, Wang WY. Phase III study of concurrent chemoradiotherapy versus radiotherapy alone for advanced nasopharyngeal carcinoma: Positive effect on overall and progression-free survival. J.Clin.Oncol. $2003 \mathrm{Feb}$ $15 ; 21(4): 631-7$.

14. Teo PML, Chan ATC. Nasopharyngeal cancer: Treatment strategy and clinical experience. Semin.Cancer Biol. 2002;12:497-504.

15. National Cancer Institute. Guidelines for reporting of adverse drug reactions; 1988. Division of cancer treatment, National Cancer Institute, Bethesda, MD.

16. World Health Organization. WHO handbook for reporting results of cancer treatment, 1979; 48. World Health Organization, Offset Publication
17. Therasse P, Arbuck SG, Eisenhauer EA, Wanders J, Kaplan RS, Rubinstein L, et al. New guidelines to evaluate the response to treatment in solid tumors. European Organization for Research and Treatment of Cancer, National Cancer Institute of the United States, National Cancer Institute of Canada. J.Natl.Cancer Inst. $2000 \mathrm{Feb}$ 2;92(3):205-16.

18. Chua DT, Sham JS, Choy D, Kwong DL, Au GK, Kwong PW, et al. Patterns of failure after induction chemotherapy and radiotherapy for locoregionally advanced nasopharyngeal carcinoma: The Queen Mary Hospital experience. Int.J.Radiat.Oncol.Biol.Phys. 2001 Apr 1;49(5):1219-28.

19. Rossi A, Molinari R, Boracchi P, Del Vecchio M, Marubini E, Nava M, et al. Adjuvant chemotherapy with vincristine, cyclophosphamide, and doxorubicin after radiotherapy in localregional nasopharyngeal cancer: Results of a 4-year multicenter randomized study. J.Clin.Oncol. 1988 Sep;6(9):1401-10.

20. Al-Sarraf M, LeBlanc M and Giri PGea. Superiority of 5-year survival with chemoradiotherapy vs radiotherapy in patients with locally advanced nasopharyngeal cancer. Intergroup 0099 Phase III study: Final report. Superiority of 5-year survival with chemoradiotherapy vs radiotherapy in patients with locally advanced nasopharyngeal cancer. Intergroup 0099 Phase III study: Final report. 2001; 20 (227a): 905.

21. Rischin D, Corry J, Smith J, Stewart J, Hughes P, Peters L. Excellent disease control and survival in patients with advanced nasopharyngeal cancer treated with chemoradiation. J.Clin.Oncol. 2002 Apr 1;20(7):1845-52.

22. Wolden SL, Zelefsky MJ, Kraus DH, Rosenzweig KE, Chong LM, Shaha AR, et al. Accelerated concomitant boost radiotherapy and chemotherapy for advanced nasopharyngeal carcinoma. J.Clin.Oncol. 2001 Feb 15;19(4):1105-10.

23. Cheng SH, Yen KL, Jian JJ, Tsai SY, Chu NM, Leu SY, et al. Examining prognostic factors and patterns of failure in nasopharyngeal carcinoma following concomitant radiotherapy and chemotherapy: Impact on future clinical trials. Int.J.Radiat. Oncol.Biol.Phys. 2001 Jul 1;50(3):717-26.

24. Hong RL, Ting LL, Ko JY, Hsu MM, Sheen TS, Lou PJ, et al. Induction chemotherapy with mitomycin, epirubicin, cisplatin, fluorouracil, and leucovorin followed by radiotherapy in the treatment of locoregionally advanced nasopharyngeal carcinoma. J.Clin.Oncol. 2001 Dec 1;19(23):4305-13.

25. Webb A, Cunningham D, Scarffe JH, Harper P, Norman A, Joffe $\mathrm{JK}$, et al. Randomized trial comparing epirubicin, cisplatin, and fluorouracil versus fluorouracil, doxorubicin, and methotrexate in advanced esophagogastric cancer. J.Clin.Oncol. 1997 Jan;15(1):261-7.

26. Preliminary results of a randomized trial comparing neoadjuvant chemotherapy (cisplatin, epirubicin, bleomycin) plus radiotherapy vs. radiotherapy alone in $\operatorname{stage} \operatorname{IV}(>$ or $=\mathrm{N} 2, \mathrm{M} 0)$ 
undifferentiated nasopharyngeal carcinoma: A positive effect on progression-free survival. International Nasopharynx Cancer Study Group. VUMCA I trial. Int.J.Radiat.Oncol.Biol.Phys. 1996 Jun 1;35(3):463-9.

27. Chan AT, Teo PM and Ngan RK et al. A phase III randomized trial comparing concurrent chemotherapy-radiotherapy with radiotherapy alone in locoregionally advanced nasopharyngeal carcinoma. A phase III randomized trial comparing concurrent chemotherapy-radiotherapy with radiotherapy alone in locoregionally advanced nasopharyngeal carcinoma. 2000; 19 (415a): 1637.

28. Chan AT, Ma BB, Lo YM, Leung SF, Kwan WH, Hui EP, et al. Phase II study of neoadjuvant carboplatin and paclitaxel followed by radiotherapy and concurrent cisplatin in patients with locoregionally advanced nasopharyngeal carcinoma: Therapeutic monitoring with plasma Epstein-Barr virus DNA. J.Clin.Oncol. 2004 Aug 1;22(15):3053-60.

29. Chan AT, Hui E, Ma B, et al. A randomized phase II study of concurrent cisplatin-radiotherapy (RT) with or without neoadjuvant chemotherapy using docetaxel (Taxotere) and cisplatin in advanced nasopharyngeal carcinoma (NPC). A randomized phase II study of concurrent cisplatin-radiotherapy (RT) with or without neoadjuvant chemotherapy using docetaxel (Taxotere) and cisplatin in advanced nasopharyngeal carcinoma (NPC). ASCO Annual Meeting Proceedings: American Society of Clinical Oncology; 2005; 23 (16S): 5544.

30. Wee J, Tan EH, Tai BC, Wong HB, Leong SS, Tan T, et al. Randomized trial of radiotherapy versus concurrent chemoradiotherapy followed by adjuvant chemotherapy in patients with american joint committee on cancer/international union against cancer stage III and IV nasopharyngeal cancer of the endemic variety. J.Clin.Oncol. 2005;23(27):6730-8.

31. Lee AW, Lau WH, Tung SY, Chua DT, Chappell R, Xu L, et al. Preliminary results of a randomized study on therapeutic gain by concurrent chemotherapy for regionally-advanced nasopharyngeal carcinoma: NPC-9901 Trial by the Hong Kong Nasopharyngeal Cancer Study Group. J.Clin.Oncol. 2005 Oct 1;23(28):6966-75.

32. Sievers KW, Greess H, Baum U, Dobritz M, Lenz M. Paranasal sinuses and nasopharynx CT and MRI. Eur.J.Radiol. 2000 Mar;33(3):185-202. 
